\title{
EVALUATION AND CHARACTERIZATION STUDY OF DUAL PULSE LASER- INDUCED SPARK (DPLIS) FOR ROCKET ENGINE IGNITION SYSTEM APPLICATION
}

\author{
Robin Osborne, ERC, Inc., Huntsville, AL, \\ Joseph Wehrmeyer, Vanderbilt University, Nashville, TN, \\ Huu Trinh, NASA MSFC Space Flight Center, Huntsville, AL, \\ and \\ James Early, Los Alamos National Laboratory, Los Alamos, NM
}

\begin{abstract}
This paper addresses the progress of technology development of a laser ignition system at NASA Marshall Space Flight Center (MSFC). Laser ignition has been used at MSFC in recent test series to successfully ignite RP1/GOX propellants in a subscale rocket chamber, ${ }^{1}$ and other past studies by NASA GRC have demonstrated the use of laser ignition for rocket engines. ${ }^{2}$ Despite the progress made in the study of this ignition method, the logistics of depositing laser sparks inside a rocket chamber have prohibited its use. However, recent advances in laser designs, the use of fiber optics, and studies of multi-pulse laser formats ${ }^{3}$ have renewed the interest of rocket designers in this state-of the-art technology which offers the potential elimination of torch igniter systems and their associated mechanical parts, as well as toxic hypergolic ignition systems. In support of this interest to develop an alternative ignition system that meets the risk-reduction demands of Next Generation Launch Technology (NGLT), characterization studies of a dual pulse laser format for laser-induced spark ignition are underway at MSFC. Results obtained at MSFC indicate that a dual pulse format can produce plasmas that absorb the laser energy as efficiently as a single pulse format, yet provide a longer plasma lifetime. In an experiments with lean $\mathrm{H} 2$ /air propellants, the dual pulse laser format, containing the same total energy of a single laser pulse, produced a spark that was superior in its ability to provide sustained ignition of fuel-lean $\mathrm{H} 2 /$ air propellants. The results from these experiments are being used to optimize a dual pulse laser format for future subscale rocket chamber tests. Besides the ignition enhancement, the dual pulse technique provides a practical way to distribute and deliver laser light to the
\end{abstract}

Copyright $\bigcirc 2003$ by the American Institute of Aeronautics and Astronautics, Inc. No copyright is asserted in the United States under Title 17, U.S. Code. The U.S. Government has a royaltyfree license to exercise all rights under the copyright claimed herein for Governmental purposes. All other rights are reserved by the copyright owner. combustion chamber, an important consideration given the limitation of peak power that can be delivered through optical fibers. With this knowledge, scientists and engineers at Los Alamos National Laboratory and CFD Research Corporation have designed and fabricated a miniaturized, first-generation optical prototype of a laser ignition system that could be the basis for a laser ignition system for rocket applications. This prototype will be tested at MSFC in future subscale rocket ignition tests.

\section{INTRODUCTION}

Concepts such as Rocket Based Combined Cycle (RBCC) and Turbine Based Combined Cycle (TBCC) are potential candidates for the Next Generation Launch Technology (NGLT) Program's future space launch vehicles. These airbreathing propulsion systems will be composed of multiple clusters of ejector rocket thrusters and small combustors throughout the vehicles. Although combustion wave ignition is considered to be a baseline ignition system for these engines, the ability of this technique to provide independently synchronized ignition sources and engine restart capability might become overly complex in its application. Although limited tests of combustion wave ignition have been performed, its implementation in actual engines could create technical issues related to ignition timing and the need to re-ignite combustors that might initially fail to ignite.

Ignition of scramjet engines within RBCC and TBCC propulsion systems is another potential issue related to future space vehicles. In scramjet engines, the mixing and subsequent burning of the supersonic airflow and injected fuel must occur within time scales that are significantly shorter than for typical, low-speed combustion devices. As a result, propellants may not be completely mixed within the scramjet combustor. Hence, very lean or rich mixtures will often pass through the scramjet 
combustor's ignition regions. So, an ability of igniting a wide range of fuel-to-air ratio is critical for the scramjet engine. Preliminary experimental and computation research ${ }^{1}$ has been shown that laser induced spark can ignite a broader range of fuel-to-air ratios than can traditional electric spark igniters as has been shown by. In addition, a broader range of flow velocities was also shown to be ignitable using laser spark ignition as compared to electrical sparks.

In the arena of conventional liquid rocket engines, large combustion chambers, such as Boeing LOX/RP-1 RS-84 rocket engines, are being considered for future Earth-to-orbit booster propulsion applications. These chambers may require baffles on the injector face to suppress combustion instability. To avoid localized pressure surges among the chamber compartments, ignition should occur simultaneously in all compartments that are formed by the baffle arrangement. Although ignition systems in existing rocket engines are operationally reliable, they use highly toxic igniter propellant, such as TEATEB, or torch igniter systems, which are composed of multiple parts. These methods may not be favorable for future engine applications.

In order to meet the need of developing innovative technologies for ultra-low-cost space transportation systems as safe and reliable as today's airliners, MSFC is working on the technology development of a laser ignition system suitable for use in rocket engine applications.

\section{Benefits of laser ignition}

A primary advantage of laser ignition is the capability of igniting propellants with laserinduced sparks at predetermined, optimal ignition locations within a combustion chamber, thereby eliminating the need for a premix chamber and propellant valves such as those used in torch igniters. The implementation of a laser ignition system would therefore eliminate ignition system mechanical parts and simplify the ignition operational sequence. With the addition of an optical multiplexing technique, the laser light could be distributed to provide multiple ignition sources that could be manipulated to occur simultaneously or sequentially, at a desired firing frequency. Figure 1 illustrates a conceptual laser ignition application. A similar ignition system could be used in RBCC, TBCC, reaction control system

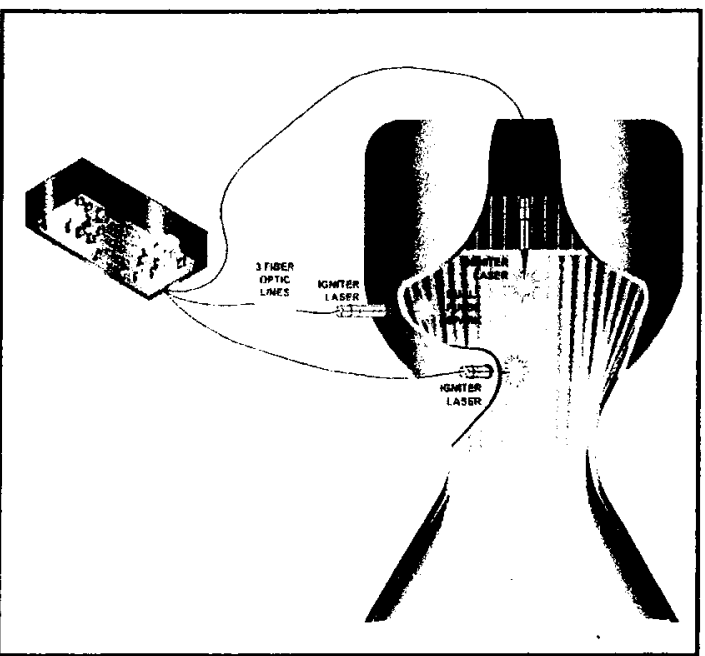

Figure 1. Conceptual laser ignition rocket application.

(RCS) rockets of future spacecrafts, and large-scale combustion chambers.

A laser ignition test series conducted by Liou $^{2}$ indicated that electromagnetic emission interference (EMI) generated from the laserinduced spark was below the permissible level for space flight. EMI is an inherent issue on many electrical spark-plug igniters. Hence, such an ignition system always requires electrical seals.

Other advantages of laser ignition include timing precision of generating a laser spark and in-flight engine restart capability.

All of these advantages are driving rationales for potentially replacing existing ignition systems, which include torch igniters and highly toxic hypergolic igniters. The goal of developing laser ignition technology is to improve reliability and operability, and reduce the operational costs of future space vehicles.

\section{Recent Work on Laser Ignition Technology at $\underline{\text { MSFC }}$}

With the collaboration of LANL and CFDRC, MSFC has performed a series of laser ignition tests in a subscale rocket combustion chamber and conducted an experimental, detailed characterization of laser sparks for optimizing ignition performance. In this effort, the dual-pulse laser-induced spark (DPLIS) ignition concept has been investigated. In DPLIS, the ignition process is accomplished by a two-step approach, using two 
distinct laser pulses, as shown schematically in Figure 2.

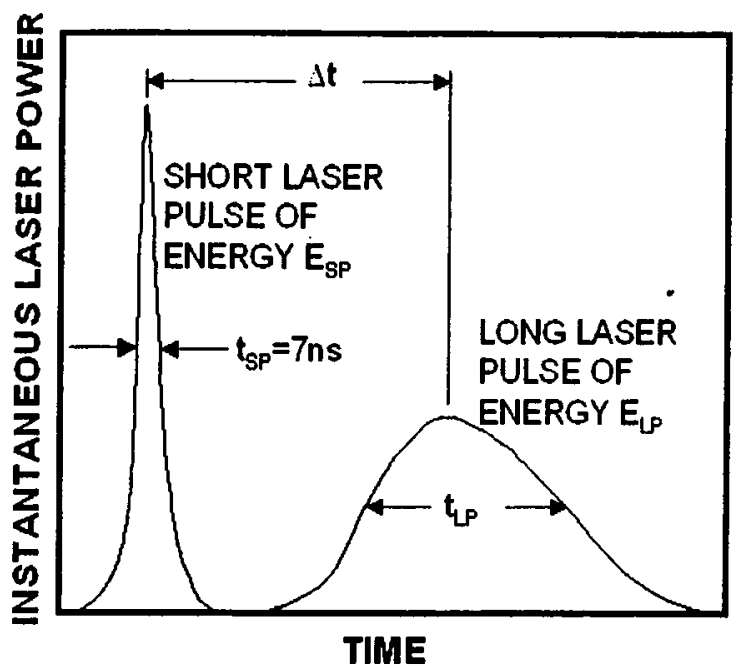

Figure 2. Schematic of the DPLIS laser format and its associated parameters.

First, a very short-duration, high-power laser pulse is focused directly into the combustible medium in order to initiate a plasma that preconditions the gas volume by maximizing the absorption of the second laser pulse. The second laser pulse provides additional photon energy and extends the plasma lifetime, enhancing the conditions for ignition. DPLIS ignition also offers the uniqueness of distributing the laser energy so that the laser light can be delivered to the combustion chamber in a practical way, using multiple fiber-optic lines. LANL and CFDRC have developed a miniaturized, first-generation prototype laser ignition system that is currently being optimized and adapted so that it can be tested with a subscale rocket chamber.

In order to optimize the laser format of this firstgeneration prototype laser ignition system for future subscale rocket chamber tests, characterization studies of the dual pulse laser format are underway. Two test series, which encompass more than two thousand tests, are being conducted with a lab-scale laser setup. In the first test series, a laser spark is deposited in a flow of air so that the energy absorbed into the plasma and the plasma lifetime can be examined. The second series is designed to study hydrogen/air ignition in an open atmosphere Hencken burner. This open environment allows easy access for diagnostics of the ignition phenomenon. The quick turn-around time of conducting experiments on this burner make it more amenable for conducting a large number of experiments for statistical analysis of the sensitivity of the test parameters.

\section{EXPERIMENTAL SETUP}

A schematic of the lab-scale laser setup is shown in Figure 3. A dual pulse custom Nd:YAG supplies $1064 \mathrm{~nm}$ wavelength, short and long laser pulses as depicted in Figure 2. The energy of the short

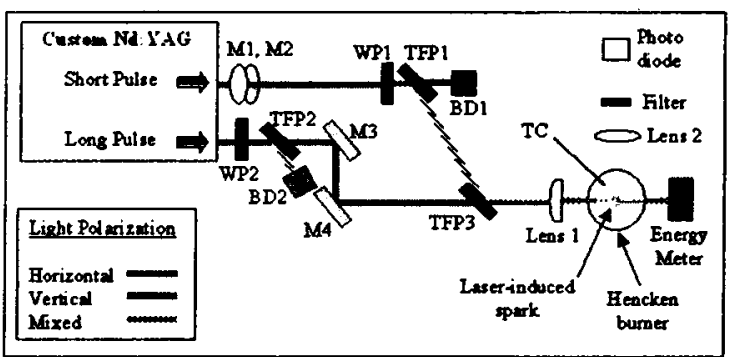

Figure 3. Schematic of the lab-scale laser setup.

pulse, $E_{S p}$, is adjustable up to $300 \mathrm{~mJ}$, while the energy of the energy of the long pulse, $E_{L P}$ is adjustable up to $350 \mathrm{~mJ}$. The temporal separation, $\Delta t$, between the two pulses is adjustable to any value, although for this work values ranging from $40 \mathrm{~ns}$ to $200 \mathrm{~ns}$ were tested. Positive values of $\Delta \mathrm{t}$ are associated with the short pulse preceding the long pulse. The temporal pulse width of the short pulse, $t_{S P}$, has a fixed value of $7 \mathrm{~ns}$ at full width half $\max$ (FWHM). Although the temporal pulse width of the long pulse, $t_{L P}$, is adjustable from $7 \mathrm{~ns}$ to $120 \mathrm{~ns}$, for this work $\mathrm{t}_{\mathrm{LP}}$ was set to $70 \mathrm{~ns}$ FWHM.

As illustrated in Figure 3, both the short and long pulse beams exit the laser horizontally polarized. Mirrors M1, M2, M3, and M4 direct the beams, while waveplates WP1 and WP2, in combination with thin film plate polarizers TFP1 and TFP2, allow adjustment of $E_{S P}$ and $E_{L P}$. Beam dumps BD1 and BD2 collect any rejected laser light. The orthogonally polarized short and long pulse beams are combined into dual pulse, collinear beams at TFP3. The dual pulse beam is focused with Lens 1 so that a laser-induced spark is formed in the openatmosphere flow of a Hencken burner.

Downstream of the laser spark, a pyroelectric joulemeter measures excess laser energy that is not absorbed in the spark. Scattered light from the laser spark is collected with Lens 2 and focused into a photo diode. A filter is placed in front of the photo diode to reject any $1064 \mathrm{~nm}$ laser light.

The Hencken burner used for this work has a 1" $\mathrm{x}$ 1 " fuel/oxidizer matrix, surrounded by a $1 / 4$ " wide 
perimeter for nitrogen shroud flow. For ignition experiments, a thermocouple was placed $31 / 4$ " above the burner exit to detect the presence of a sustained flame.

\section{RESULTS}

\section{Characterization of Laser Sparks}

At MSFC, detailed studies of laser sparks in air were conducted to characterize and understand the differences in single and dual pulse techniques. This characterization included how the duration of the spark and absorption of the laser energy into the spark are affected by the input laser energies, $E_{S P}$ and $E_{L P}$, the focal length of the lens (shown as Lens 1 in Figure 3), and in the case of dual pulse, $\Delta t$ between the two pulses. Figure 4 illustrates how both the input pulse energy and focal length of the lens affect how efficiently the laser energy is absorbed into the spark. These are important

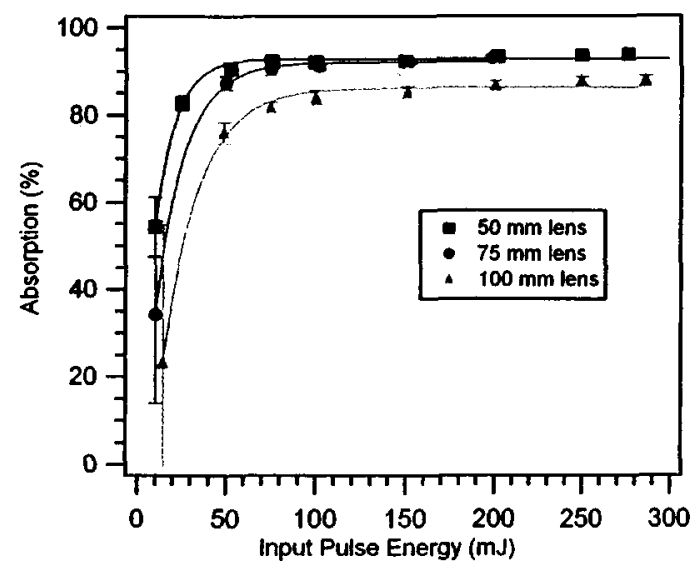

Figure 4. Percent absorption of a single laser pulse in air, as a function of input laser energy and lens focal length.

considerations, regardless of whether the final design is single or dual pulse. These results indicate that for a typical q-switched Nd:YAG laser operated at $1064 \mathrm{~nm}$, a laser energy input vale of $100 \mathrm{~mJ}$ or greater closely approaches the maximum absorption value possible for the three focal length lenses studied. These focal lengths represent what are considered to be realistic distances for location of the spark with respect to the combustion chamber wall. For example, a $100 \mathrm{~mm}$ focal length lens would allow the spark to be positioned nearly $100 \mathrm{~mm}$ (4") from the chamber wall where the laser igniter is mounted. The data in Figure 4 implies that the further the spark reaches into the chamber, the less efficiently the laser energy is absorbed into the spark. This would simply mean that slightly more input energy might be required for applications that require a longer reach of the spark into the combustion chamber.

Figure 5 illustrates, for a spark produced by a single laser pulse in air, how the spark duration, also referred to as the plasma lifetime, is extended with increasing input pulse energy. For this work, the plasma lifetime is defined as the total time

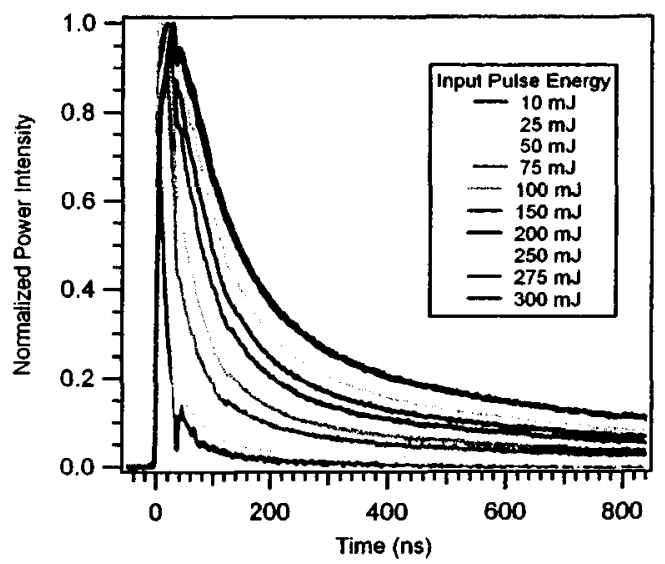

Figure 5. Traces of plasma temporal profiles as a function of input pulse energy for a single laser pulse focused with a $50 \mathrm{~mm}$ lens.

between the limits where the plasma power intensity rises to $15 \%$ of its peak value and then falls to $15 \%$ of that peak value. As shown in Figure 6, both the input laser pulse energy and lens focal length strongly influence the plasma lifetime. Higher input pulse energy as well as shorter focal length lenses extends the plasma lifetime.

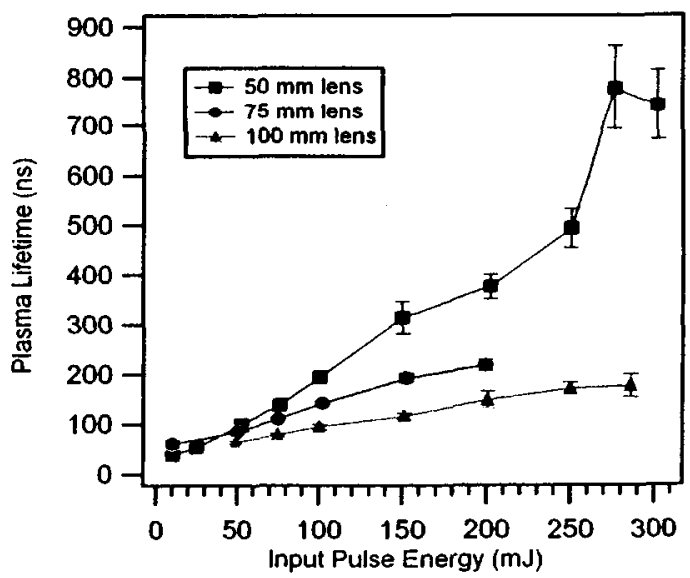

Figure 6. Plasma lifetime of a single laser pulse in air, as a function of input laser energy and lens focal length.

In general, it is believed that a longer plasma lifetime should be beneficial to the onset of ignition since it represents the time period during 
which free electrons exist and collide with molecules in order to produce chemical radicals. These chemical radicals initiate the chainbranching reactions that lead to ignition.

In experiments carried out with dual pulse sparks in air, for each of the three lenses tested it was found that for temporal separations of 40-200 ns a dual pulse with $100 \mathrm{~mJ}$ in both the first and second pulse (200 mJ total energy) absorbed energy into the spark as well as or nearly as well as a $200 \mathrm{~mJ}$ single pulse spark (see Figure 7). However, the second pulse had a pulse with of about $70 \mathrm{~ns}-\mathrm{a}$ factor of 10 longer than the first pulse and also a factor of 10 longer than the $200 \mathrm{~mJ}$ single pulse.

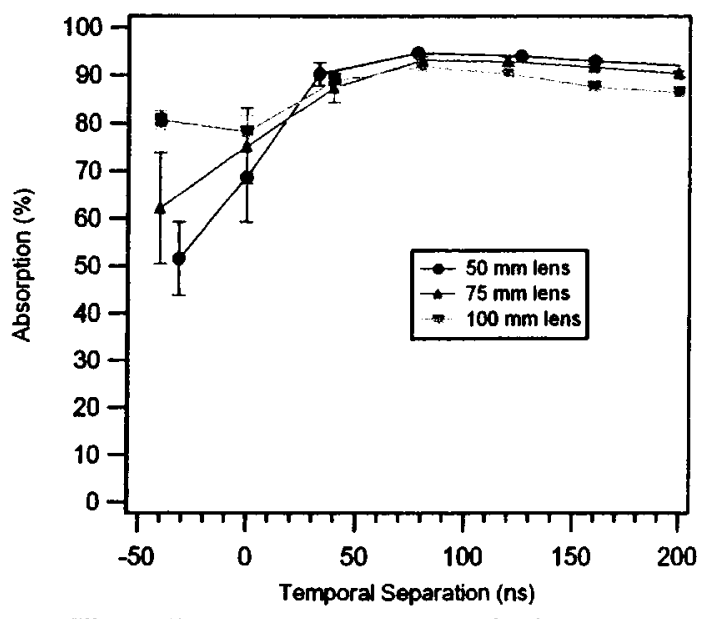

Figure 7. Percent absorption of a $200 \mathrm{~mJ}(100 \mathrm{~mJ}$ in both the short and long pulses) dual laser pulse into a spark in air, as a function of temporal separation and lens focal length.

The $200 \mathrm{~mJ}$ dual pulse spark therefore provides nearly equivalent absorption to the $200 \mathrm{~mJ}$ single pulse, but provides a factor of two reduction in peak power of the first pulse and a factor of 20 reduction in the second pulse. Another observation of the dual pulse format, as shown in Figure 7, is that in some cases it extends the plasma lifetime beyond that which was observed for single pulse sparks of equivalent energy. For example, in Figure 6, for the case where a $50 \mathrm{~mm}$ lens was used, plasma lifetimes are extended by as much as $100 \mathrm{~ns}$ for some values of temporal separation. An interesting result as seen in Figure 8 is that dual pulses with equivalent energies and equivalent absorption can generate plasmas that have substantially different lifetimes. This is the case of a dual pulse with $50 / 100 \mathrm{~mJ}$ and $100 / 50 \mathrm{~mJ}$. These two pulse formats each have similar absorption characteristics for a given lens, but as can been seen in Figure 8, the 50/100 mJ format can produce plasmas that are about $150 \mathrm{~ns}$ longer for certain

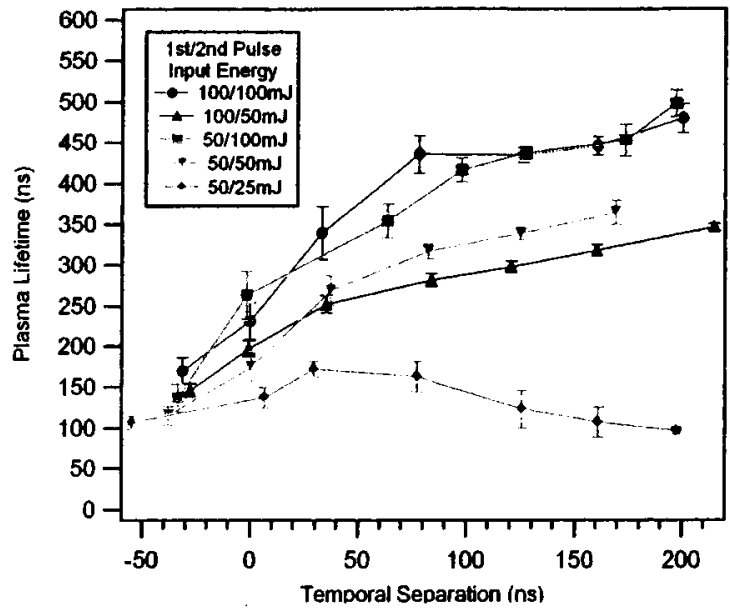

Figure 8. Plasma lifetime of a dual pulse laser spark focused with a $50 \mathrm{~mm}$ lens, as a function of input laser energy and temporal separation.

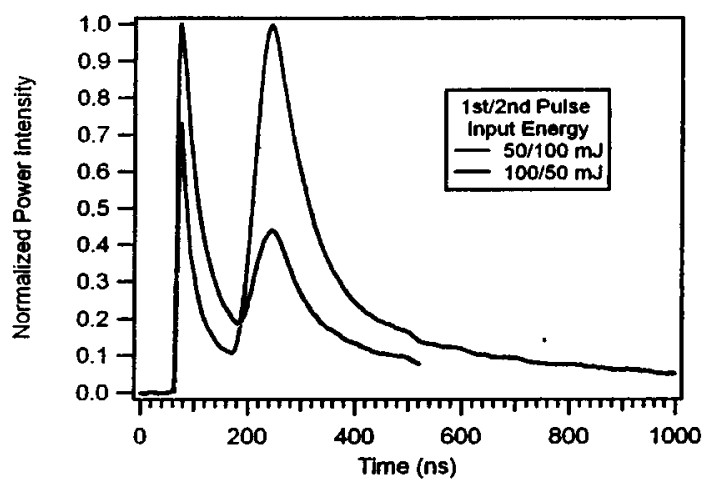

Figure 9. Profiles of two plasmas, each produced by dual pulses of the same total energy, both focused by $50 \mathrm{~mm}$ lenses.

temporal separations. The temporal traces for the two plasmas are displayed in Figure 9.

\section{Hencken Burner Ignition Tests}

Ignition tests using laser sparks were conducted in the Hencken burner ${ }^{4}$ by flowing a mixture of $\mathrm{H}_{2}$ /air. A very lean mixture of $\Phi=0.26$ was selected in order to create an environment in which ignition might not be easily achieved. This environment provides a higher sensitivity to the selected parameters of laser energy, single or dual pulse, and in the case of dual pulse, the $\Delta t$ between the two pulses.

For the ignition tests, the laser beam was focused with a $75 \mathrm{~mm}$ lens into a spark centered $1.2 \mathrm{~cm}$ above the exit of the Hencken burner. A thermocouple centered $31 / 4$ " above the Hencken 
burner was used to detect sustained ignition events. For each laser format studied, the test was repeated 30 times. Ignition was attempted with single pulse laser sparks having $100,150,200$, and $250 \mathrm{~mJ}$. Out of the thirty tests conducted for each of these single pulse formats, none resulted in sustained ignition. Figure 10 shows the ignition data resulting from a dual pulse containing $150 \mathrm{~mJ}$ in the first pulse and $50 \mathrm{~mJ}$ in the second pulse, with seven different $\Delta t$ formats. As expected, a $\Delta t$ value of $-40 \mathrm{~ns}$ resulted in no sustained ignition events.

Likewise, no ignition events were recorded for $\Delta \mathrm{t}=$ $0 \mathrm{~ns}$. However, the four other $\Delta \mathrm{t}$ formats tested did result in successful ignition events, with a $\Delta t=80$ ns format igniting the lean mixture in 20 of the 30 tests.

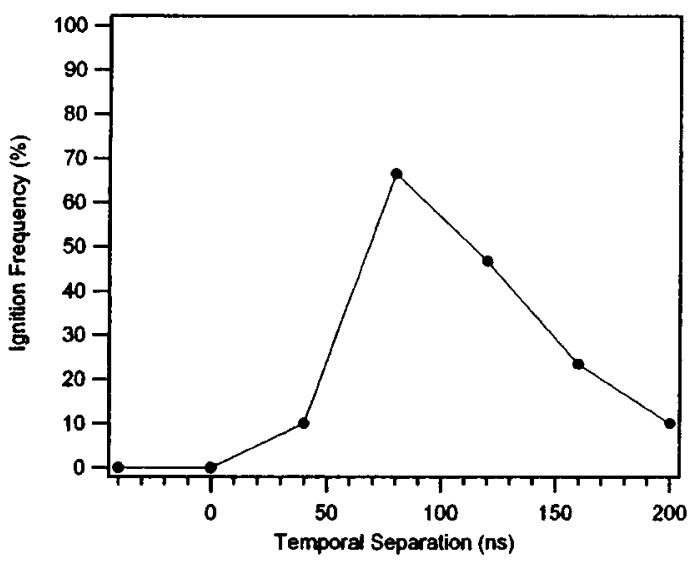

Figure 10. Frequency of ignition events as a function of $\Delta t$ for a dual pulse spark $(150 \mathrm{~mJ}$ in the first pulse and $50 \mathrm{~mJ}$ in the second pulse) focused with a $75 \mathrm{~mm}$ lens.

\section{CONCLUSIONS}

A detailed study is underway at MSFC to characterize lasers sparks and understand the differences between single and dual pulse laser sparks and their ability to ignite propellants. A study of laser sparks produced in air show that a dual pulse format can produce plasmas that absorb the laser energy as efficiently as a single pulse format, yet provides a longer plasma lifetime. Experimental results obtained at MSFC reveal that a dual pulse laser format, containing the same total energy of a single laser pulse, produces a spark that is superior in its ability to provide sustained ignition of fuel-lean $\mathrm{H} 2$ /air propellants. Without further testing, it is unclear whether the enhancement of the dual pulse format is actually attributed to the longer plasma lifetimes. There is speculation that the dual pulse format might also more efficiently couple the laser energy into the plasma with fewer energy losses into other energy modes such as radiation and shock waves, phenomena not measurable with the instrumentation used in the current experiments.

Besides the ignition enhancement, the dual pulse technique provides a practical way to distribute and deliver laser light to the combustion chamber, an important consideration given the limitation of peak power that can be delivered through optical fibers.

Future work planned for this study includes using an intensified charge-coupled device (ICCD) to study the spatial profile of single and dual pulse sparks and track the formation of the combustion kernel associated with ignition. This should give more insight into the differences of these two laser ignition techniques.

Lab-scale laser ignition tests are also planned for a subscale $\mathrm{GH} 2 / \mathrm{GOX}$ rocket chamber. A

miniaturized, first-generation laser ignition system prototype will also be tested in subscale rocket chamber tests of RP1/GOX as well as GH2/GOX.

\section{ACKNOWLEDGEMENTS}

The authors gratefully acknowledge the assistance of Matthew Thomas and John Bossard at CFDRC, Paul Dumbacher at MSFC and Domenic Santavicca at Pennsylvania State University.

\section{REFERENCES}

1. Trinh, H. P., Early, J. W., Osborne, R. J., Thomas, M. E., and Bossard, J. A., "Technology Development of a Fiber Optic-Coupled Laser Ignition System for Multi-Combustor Rocket Engine," paper presented at the $14^{\text {th }}$ Annual Symposium on Propulsion, Penn State Propulsion Engineering Research Center, University Park, PA, December 10-11, 2002.

2. Liou, L. C., "Laser Ignition in Liquid Rocket Engines," paper presented at the $30^{\text {th }}$ AIAA/SAE/ASME/ASEE Joint Propulsion Conference, Indianapolis, IN, June 27-29, 1994.

3. Thomas, M. E., "Multi-Pulse Laser Ignition for UItra Cold Starting of TurboShaft Engines," Final Report (SBIR Phase 
I Project), U. S. Army Contract:

DAAH10-98-C-0008, August, 1998.

4. Burner purchased from Research

Technologies, P. O. Box 384, Pleasanton,

CA., Ken Hencken, President. 